\title{
INFLUENCE OF NUMBER AND THICKNESS OF BLADES ON THE PERformanCe OF CENTRIfUgal FANS
}

\author{
Vaclav Dvorak \& Anas F. A. Elbarghthi
}
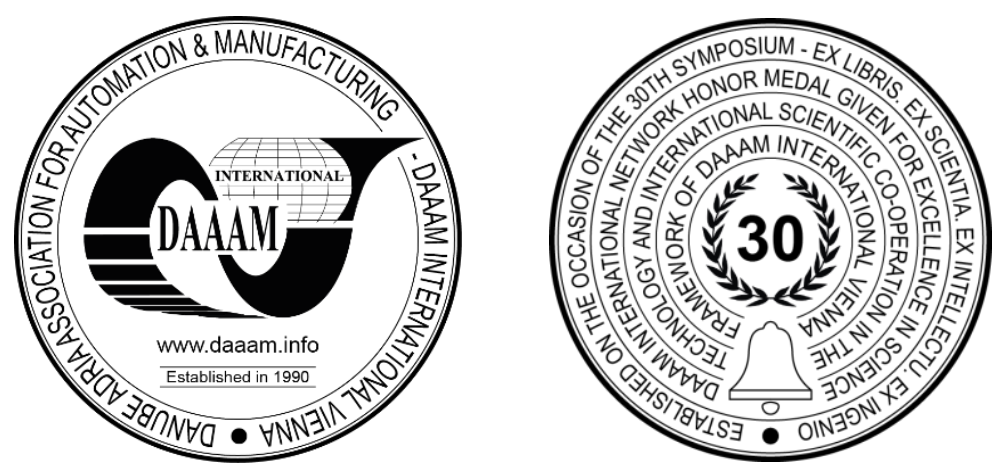

This Publication has to be referred as: Dvorak, V[aclav] \& Elbarghthi, A[nas] (2020). Influence of Number and Thickness of Blades on the Performance of Centrifugal Fans, Proceedings of the 31st DAAAM International Symposium, pp.0724-0727, B. Katalinic (Ed.), Published by DAAAM International, ISBN 978-3-902734-29-7, ISSN 1726-9679, Vienna, Austria

DOI: $10.2507 / 31$ st.daaam.proceedings. 100

\begin{abstract}
The article deals with the research of the influence of the number of blades on the performance curve of small highpressure centrifugal fans. The classical Euler equation for fans predicts that as the number of blades increases, the socalled loss due to inter-blade circulation will decrease. However, this is in direct contrast to experiments performed on a series of small fan wheels with backward curved blades, as the performance of the fans is only negligibly affected by the number of blades. The analysis performed in the article shows that the circulation between the blades does not cause a loss in terms of energy dissipation, but a change in the velocity diagram. A modified Euler equation is presented, which includes not only the effect of the number of blades, but also the effect of non-zero blade thickness. It is explained how the additional blades adversely affect the resulting fan characteristic. The resulting analytical model shows better agreement with the results of experiments and numerical calculations.
\end{abstract}

Keywords: Centrifugal fans; radial high-pressure fans; blade thickness; number of blades.

\section{Introduction}

The work's motivation is developing a new generation of motor respirators for air purification intended for the filtration of pollutants in the form of gases, vapors and particles, or biological pollution. These high-performance units guarantee sufficient protection of the wearer even in environments of heavy industry, chemical industry, laboratories and pharmaceutical industry. To help people breathe through a filter, a fan is used to drive air flowing through personal systems and respiratory protection units. The performance of a centrifugal fan depends on many parameters, including the outer diameter $d_{2}$, the width of the wheel $b_{2}$, the design of the blades, in particular the outlet angle of the blade $\beta_{2}$ and, last but not least, the number of blades $z$. A large number of blades tend to reduce flow separation and loss via interblade circulation, but also restrict inlet airflow and also increase friction losses. It is generally assumed that it is difficult to predict the optimal number of blades using theoretical methods, because the blade number has little effect on the fan characteristics [1], [2]. For these reasons, researchers are focusing either on experimental research into the effect of the number of blades on the fan characteristics [3] or on numerical calculations [4]. However, these works lack any analysis. This work aims to answer the question why the number of blades actually has such a small effect on the operation of the fan and what will be the effect of blade thickness on the performance of very small fans. 


\section{Methods}

The geometry of the investigated impellers can be seen from Fig. 1 on the left. Radial wheels with curved blades were investigated both experimentally and by numerical and theoretical methods. All impellers had a diameter $d_{2}=60 \mathrm{~mm}$ and were made by 3D printing technology [5]. The fan was controlled to maintain a constant speed [6].
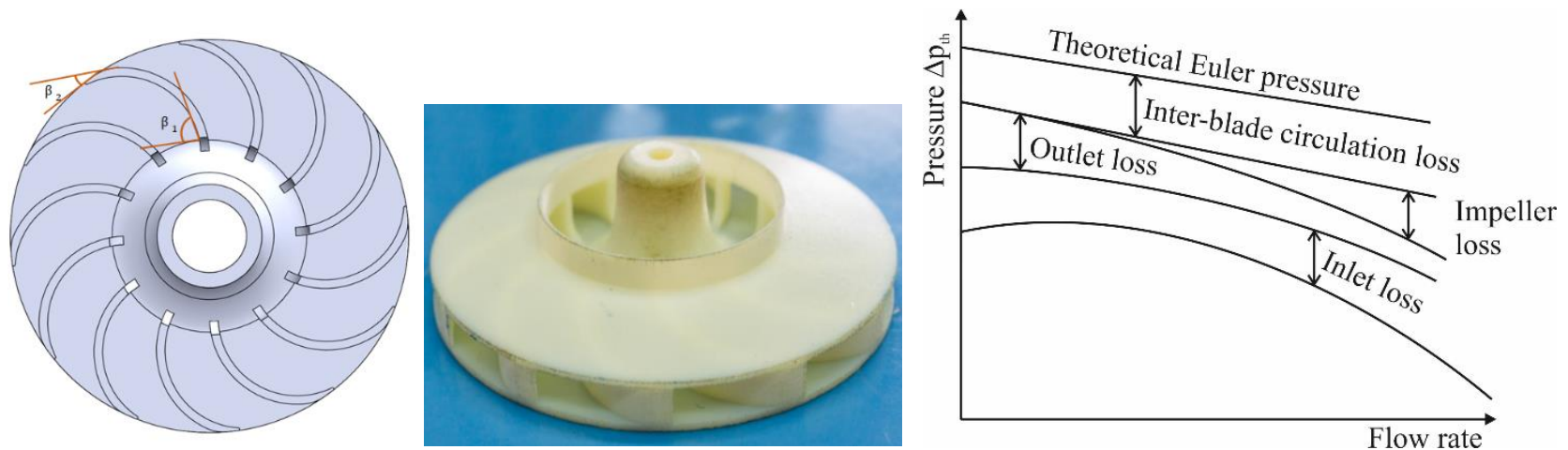

Fig. 1. Investigated impeller (on the left), wheel manufactured by 3D printing technology (in the middle) and theoretical and real fan performance curve (on the right).

Although the one-dimensional method is only indicative, it provides information to understand the events that occur in the fans and can be used to quickly design the basic dimensions of a new fan. Theoretical increase of the total pressure $\Delta p_{t h}$ in a centrifugal fan is described by the Euler's pump equation [7]

$$
\frac{\Delta p_{t h}}{\rho}=c_{2 u} u_{2}=u_{2}^{2}\left(1-\frac{c_{2 m}}{u_{2}} \frac{1}{\tan \beta_{2}}\right)
$$

in which $\rho$ is the density of the medium (air), $u_{2}$ is the circumferential or blade velocity, $c_{2 u}$ is the component of the total velocity in the circumferential direction, $u_{2 m}$ is the component of the total velocity in the predominant flow direction, here in the radial direction, and $\beta_{2}$ is the blade exit angle. The relation gives the theoretical working characteristic for lossless flow, see Fig. 1 on the right. In order to get a real characteristic, it is necessary to take into account individual losses, as did, for example, Purnell [7]. Losses can be divided into several areas based on the area in which they occur, i.e. input losses, impeller losses and output losses. The mentioned losses are further composed of other partial losses. E.g. the output loss is due to the imbalance of the velocity profile, or the loss by sudden expansion. Input losses are caused by a change in direction or an acceleration of the current at the impeller inlet. Impeller losses are caused by friction in the duct and flow separation. A special "loss" is the so-called inter-blade circulation described in Fig. 2 on the left.

The inter-blade circulation is caused by the inertia of the fluid that does not rotate with the impeller, resulting in a local vortex relative to the rotating wheel. As can be seen from the figure, the vortex causes the outlet absolute velocity vector $c_{2}$ to rotate in the opposite direction, which causes the outlet angle to decrease from $\beta_{2}$ to $\beta_{2}$ and decrease the blade component of the total velocity from $c_{2 u}$ to $c_{2 u}$. As can be seen from Euler's equation (1), decreasing $c_{2 u}$ causes a decrease in the theoretical pressure $\Delta p_{t h}$.
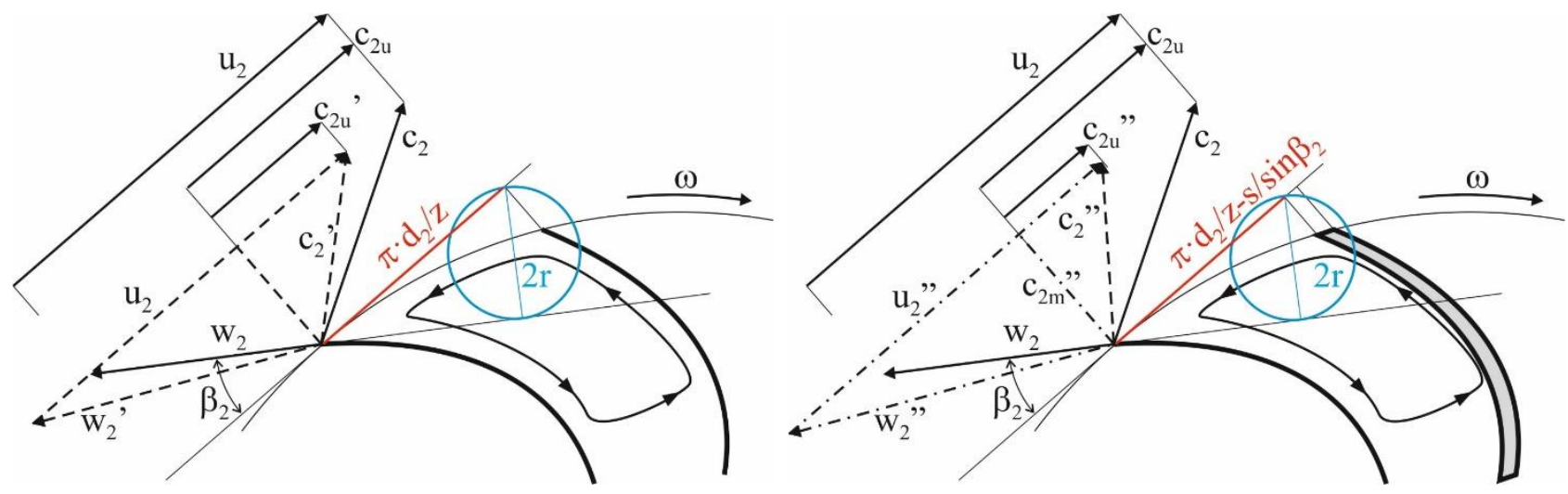

Fig. 2. Inter-blade circulation loss (on the left), the effect of blade thickness (on the right).

In essence, this decrease of $\Delta p_{t h}$ is not a loss, as it is not a way of dissipating mechanical energy, but a change in the velocity diagram. The inter-blade circulation "loss" is expressed as 


$$
\frac{\Delta p_{\text {blade }}}{\rho}=\mathrm{u}_{2} r \omega
$$

where $r$ is the radius of the imaginary circle between two blades, see Fig. 2 on the left, and is defined as

$$
r=\frac{\pi d_{2} \sin \beta_{2}}{2 z},
$$

where $z$ is number of blades. The modified Euler's pump equation including the effect of the inter-blade circulation is

$$
\frac{\Delta p_{t h}}{\rho}=u_{2}^{2}\left(1-\frac{c_{2 m}}{u_{2}} \frac{1}{\tan \beta_{2}}-\frac{\pi \sin \beta_{2}}{z}\right) .
$$

As we can see, the above relation does not respect the thickness of the blade in any way.

So let's look at how a non-zero blade thickness affects the velocity diagram. As can be seen from Fig. 2 on the right, a blade reduces the radius of the local vortex by its thickness according to the relation

$$
r=\frac{\pi d_{2} \sin \beta_{2}}{2 z}-\frac{s}{2},
$$

where $s$ is the blade thickness. The thickness of blades also limits the flow cross-section, so that the velocity $c_{2 m}$ is increased. According to Fig. 2 on the right, this causes the output relative speed $w_{2}$ to be extended and $c_{2 u}$ to be reduced. The final Euler's pump equation, while the inter-blade circulation "loss" and the effect of blade thickness are included, is

$$
\frac{\Delta p_{t h}}{\rho}=u_{2}^{2}\left[1-\frac{Q}{\left(\pi d_{2}-\frac{z s}{\sin \beta_{2}}\right) b_{2} u_{2}} \frac{1}{\tan \beta_{2}}-\frac{\pi \sin \beta_{2}}{z}+\frac{s}{d_{2}}\right] \text {. }
$$

\section{Results}

The resulting theoretical characteristics of the radial fan depending on the number of blades are shown in Fig. 3. The working curve according to (4) is shown on the left. The positive effect of the blades on reducing the loss of inter-blade circulation is very noticeable. If the blade thickness is taken into account, Fig. 3 on the right, the effect of the additional blades is sporadic. As shown in Fig. 2 on the right and in (6), the non-zero blade thickness has a similar effect as the interblade circulation. So another blade can even reduce the pressure gained. The negative effect of the blade will grow with the $s / d_{2}$ ratio. It is worth noting that due to the existence of boundary layers, the negative effect of the number of blades will not be negligible even for larger fans with thinner blades.
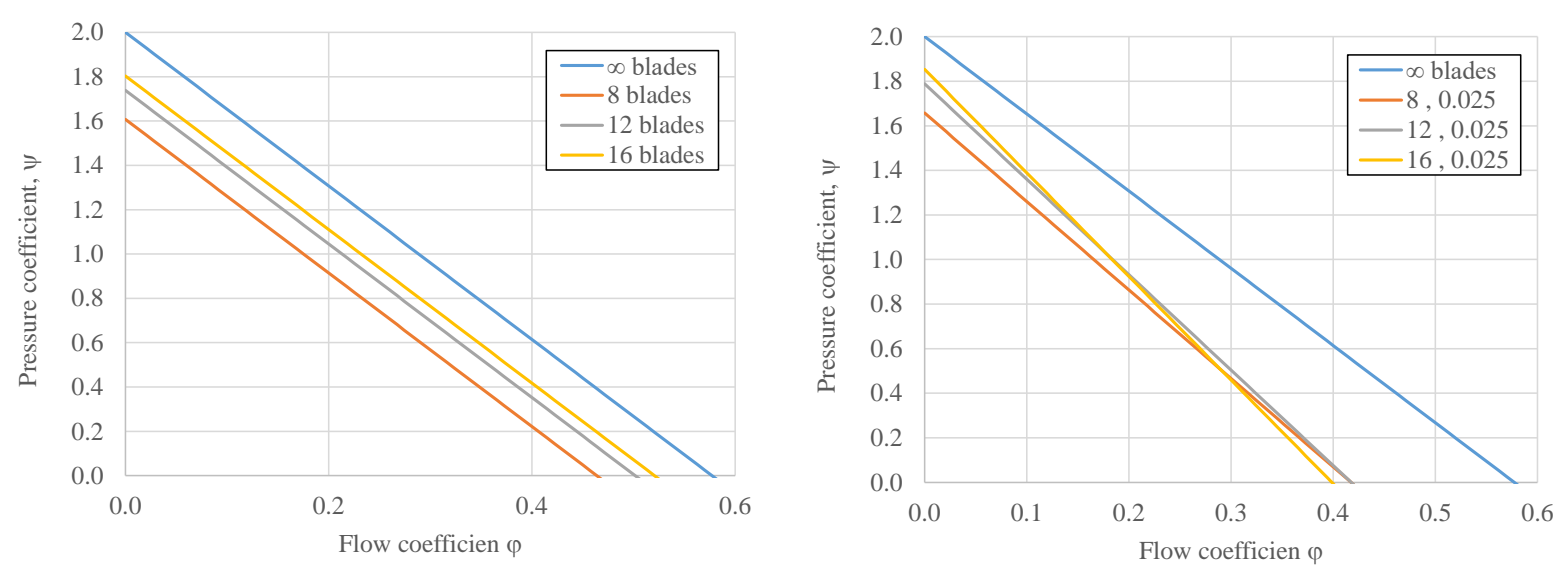

Fig. 3. Theoretical fan performance curve without (on the left) and with (on the right) effect of blade thickness.

The results of the modified Euler's equation (6) are consistent with both the experiments [8] and the results of the numerical calculations [10] in Fig. 4 on the left, where the working curves of the fan with 10 to 14 blades are plotted. Although the results of the individual methods differ, it is clear that the effect of the number of blades is negligible and shows no trend. In Fig. 4 on the right are the results of the theoretical method with the incorporation of all losses according to the work [7] and equation (6). The influence of the number of blades is noticeable especially for the low flow, i.e. in the part of the characteristics where the flow at the outlet of the impeller is no longer ideal due to the high back pressure. In this area, the theoretical method loses its validity due to the backflow into the impeller. 

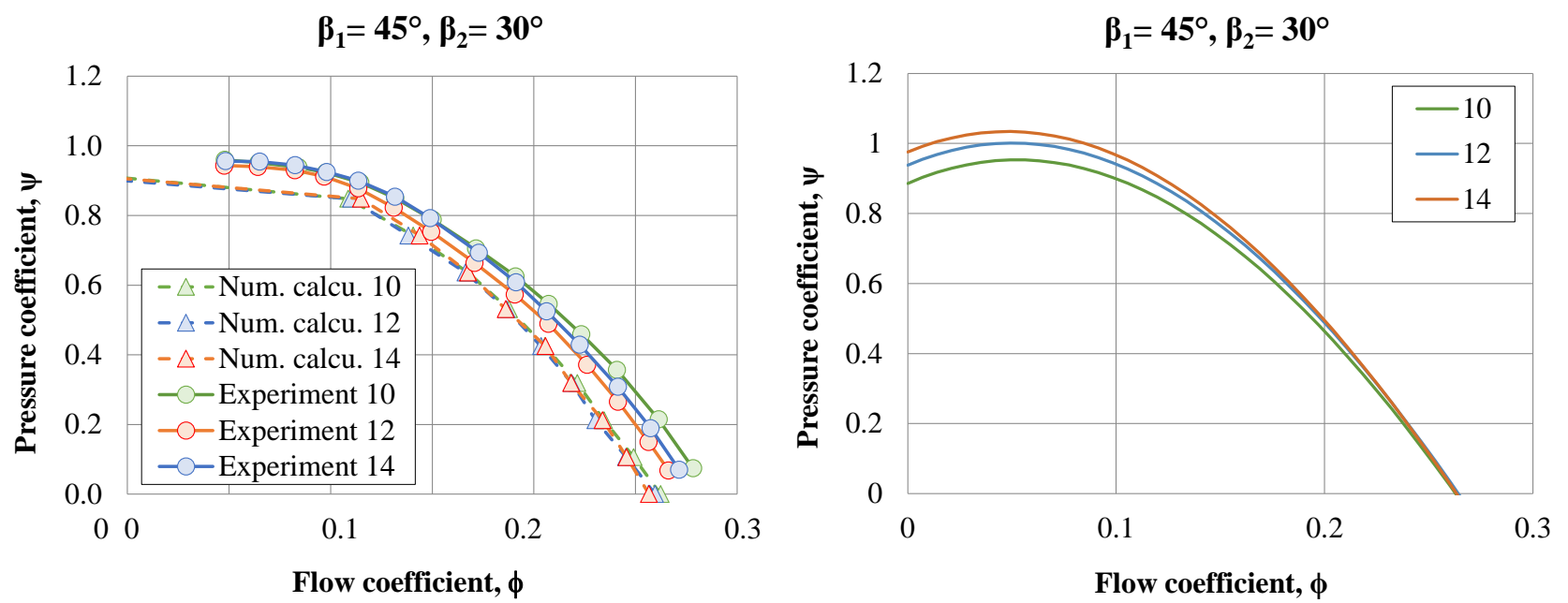

Fig. 4. Influence of blade number on the performance curve of the centrifugal fan; experimental [8] and numerical [9] (on the left) and theoretical (on the right).

\section{Conclusions}

The aim of the work was to solve the discrepancies between the theoretically predicted influence of the number of blades on the performance of the centrifugal fan and the results of experimental data. To address this, an analysis of the effect of blade thickness on the velocity diagrams was performed. This analysis has shown that the higher number of blades increases the achieved pressure due to the reduction of the inter-blade circulation, but the effect of non-zero blade thickness is negative. It can be assumed that the thickness of the fluid boundary layers on the blades will have the same effect as the thickness of the blade itself. The results of the modified theoretical fit very well with the results of numerical and experimental research on impellers with 10,12 and 14 blades. In the future work, the improvement of the 1D method will be solved, especially with regard to the influence of the blade wall thickness on the friction in the channel between the blades.

\section{Acknowledgments}

This paper was created in the framework of the project "Applied research in the field of the new generation of personal protective equipment for the demands of joint rescue service" no. VI20172020052, supported by the Ministry of Interior of the Czech Republic.

\section{References}

[1] Gülich, J.F. (2010). Centrifugal Pumps (2nd ed.). Berlin: Springer-Verlag. ISBN 978-3-642-12823-3.

[2] Hunter, J. S. (1996) A design philosophy for centrifugal fans, Master thesis, Loughborough University, Great Britain.

[3] Mwinuka, T. (2016) Experimental Determination of the Effect of Number of Impeller Blades on the Air Flow Rate and Power Consumption of Centrifugal Blowers. American Scientific Research Journal for Engineering, Technology, and Sciences (ASRJETS), Vol. 17, No 1, pp 81-88. ISSN (Online) 2313-4402.

[4] Li Zhang \& Yingzi Jin. (2011). Effect of Blade Numbers on Aerodynamic Performance and Noise of Small Axial Flow Fan. Advanced Materials Research, Vol. 199-200, pp 796-800, ISSN: 1662-8985.

[5] Dvořák, V.; Votrubec, R.; Šafka, J. \& Kracík, J. (2018). Experimental investigation of centrifugal fans for personal protection equipment - effect of used 3D printing technologies, EPJ web of Conferences, ISSN: 2101-6275.

[6] Votrubec, R. \& Buchta, J. (2019). Air Flow Control of Centrifugal Fans for Personal Protection Equipment, Proceedings of the 30th DAAAM International Symposium, pp.0268-0275, B. Katalinic (Ed.), Published by DAAAM International, ISBN 978-3-902734-22-8, ISSN 1726-9679, Vienna, Austria

[7] Purnell, John G. (1973). Prediction of Axial and Centrifugal Fan Characteristics for Large Surface Effect Vehicles. David W. Taylor Naval Ship Research and Development Center Annapolis MD. AD0912307

[8] Moro, G. (2019) Experimental Investigation of fans for personal protective equipment, Master thesis, Technical University of Liberec, Czech Republic.

[9] Lampa, J. (2019) Design of a fan for personal protective equipment, Master thesis, Technical University of Liberec, Czech Republic. 